\title{
The feasibility of a single-blinded fast-track pragmatic randomised controlled trial of a complex intervention for breathlessness in advanced disease
}

\author{
Morag C Farquhar*1, Irene J Higginson ${ }^{2}$, Petrea Fagan ${ }^{3}$ and Sara Booth ${ }^{3}$
}

\begin{abstract}
Address: ${ }^{1}$ General Practice and Primary Care Research Unit, Dept Public Health \& Primary Care, University of Cambridge, Institute of Public Health, Robinson Way, Cambridge, CB2 OSR, UK, ${ }^{2}$ Department of Palliative Care, Policy \& Rehabilitation, King's College London, Weston Education Centre, Cutcombe Rd, London, SE5 9RJ, UK and ${ }^{3}$ Breathlessness Intervention Service, Box 193, Addenbrooke's Hospital, Cambridge University Hospitals' NHS Foundation Trust, Hills Road, Cambridge, CB2 0QQ, UK
\end{abstract}

Email: Morag C Farquhar* - mcf22@medschl.cam.ac.uk; Irene J Higginson - Irene.higginson@kcl.ac.uk; Petrea Fagan - petrea.fagan@addenbrookes.nhs.uk; Sara Booth - sara.booth@addenbrookes.nhs.uk

* Corresponding author

Published: 7 July 2009

BMC Palliative Care 2009, 8:9 doi:10.1/86/1472-684X-8-9
Received: 6 March 2009

Accepted: 7 July 2009

This article is available from: http://www.biomedcentral.com//472-684X/8/9

(c) 2009 Farquhar et al; licensee BioMed Central Ltd.

This is an Open Access article distributed under the terms of the Creative Commons Attribution License (http://creativecommons.org/licenses/by/2.0), which permits unrestricted use, distribution, and reproduction in any medium, provided the original work is properly cited.

\begin{abstract}
Background: The Breathlessness Intervention Service is a novel service for patients with intractable breathlessness regardless of aetiology. It is being evaluated using the Medical Research Council's framework for the evaluation of complex interventions. This paper describes the feasibility results of Phase II: a single-blinded fast-track pragmatic randomised controlled trial.

Methods: A single-blinded fast-track pragmatic randomised controlled trial was conducted for patients with chronic obstructive pulmonary disease referred to the service. Patients were randomised to either receive the intervention immediately for an eight-week period, or receive the intervention after an eight-week period on a waiting list during which time they received standard care. Outcomes examined included: response rates to the trial; response rates to the individual questionnaires and items; comments relating to the trial functioning made during interviews with patients, carers, referrers and service providers; and, researcher fieldwork notes.

Results: 16 of the 20 eligible patients agreed to participate in a recruitment visit (16/20); 14 respondents went on to complete a recruitment visit/baseline interview. The majority of those who completed a recruitment visit/baseline interview completed the RCT protocol (I3//4); 12 of their carers were recruited and completed the protocol. An unblinding rate of $6 / 25$ respondents (patients and carers) was identified. Missing data were minimal and only one patient was lost to follow up. The fast-track trial methodology proved feasible and acceptable. Two of the baseline/outcome measures proved unsuitable: the WHO performance scale and the Schedule for the Evaluation of Individual Quality of Life-Direct Weighting (SEIQoL-DW).

Conclusion: This study adds to the evidence that fast-track randomised controlled trials are feasible and acceptable in evaluations of palliative care interventions for patients with non-malignant conditions. Reasonable response rates and low attrition rates were achieved. Further, with adequate preparation of the research and randomisation teams, clinicians, and responders, and effective liaison with the clinicians, single-blinding proved possible. Methods were identified to reduce unblinding through careful attention to the type of data collected at unblinded measurement points; the content of interviews should be carefully considered when designing blinded-trial protocols.
\end{abstract}

Trial registration: Clinical Trials.gov NCT007/ I438 


\section{Background}

Intractable breathlessness is common in advanced disease, both malignant and non-malignant. In chronic obstructive pulmonary disease (COPD) and heart failure it is nearly universal by the time of death, its prevalence reaching $90-95 \%$ and $60-88 \%$ respectively in the advanced stages [1]. Recent advances in the palliation of breathlessness include non-pharmacological intervention services to reduce or contain the severity of the sensation. Formally evaluated services have had positive outcomes in terms of reduction in distress caused by breathlessness, increased functioning and quality of life [2-5], however these services focused solely on patients with malignant disease and their evaluations were methodologically limited (e.g. only two were randomised controlled trials (RCTs) and published outcomes were limited to patient outcomes only). These were complex interventions which are notoriously difficult to evaluate [6].

The Medical Research Council's Framework for the Development and Evaluation of RCT for Complex Interventions to Improve Health [6] was developed to combat the unique challenge of evaluating complex interventions by building a continuum of increasing evidence to support the effective development of complex interventions through robust methodology. It was described as the current method of choice for assessing complex interventions for breathlessness by the 2005 Medical Research Council (MRC) Clinical Trials Unit/Cicely Saunders Foundation 'Improving Research Methodology in Breathlessness' meeting [7].

The Breathlessness Intervention Service (BIS) Service at Addenbrooke's NHS Trust is being evaluated using the MRC framework [6]. BIS aims to manage the symptom of intractable breathlessness in patients with disease of any aetiology (malignant and non-malignant) using a rehabilitative approach. Interventions include evidence-based non-pharmacological interventions (psychological, social and physical), palliative care input (e.g. end of life issues, psychosocial issues, family concerns) and pharmacological review. Thus BIS seeks to enhance the self-management of breathlessness. Uniquely, care is flexibly located preferentially in patients' own homes, or in a clinic if more appropriate. Referrals come from medical specialists, GPs and allied health professionals (with medical consent). At Phase II BIS was staffed by a clinical specialist physiotherapist and palliative care consultant.

The MRC framework has recommended RCTs as the gold standard for evaluating services [6], however conducting RCTs within palliative care is challenging because of difficulties with recruitment, due to acceptability, and attrition, associated with increasing morbidity and death [8]. Following the experience of Higginson et al (2006 \&
2008) $[9,10]$, a fast-track RCT model was selected to address these difficulties. This design has previously been attempted in palliative care by McWhinney et al (1994) [11] to evaluate a palliative care home support team but this single-blinded trial failed to recruit an adequate sample size due to problems with attrition and inaccurate prognostication; it is unclear what diagnoses the intervention was targeted at, but it was likely to have been patients with malignancies. The recent successful palliative care fast-track RCT by Higginson et al (2006 \& 2008) evaluated a palliative care service for people affected by multiple sclerosis, however the authors made no attempt at blinding $[9,10]$. Hart et al $(2008)$ describe the 'impossibility' of double-blinding in experience-based trials, but stress the importance of single-blinding the investigator where possible [12]. Thus the Phase II study reported here sought to test the feasibility of single-blinding in a fast-track pragmatic RCT of BIS versus standard care for patients with a different non-malignant disease (COPD) and their informal carers. Results of the earlier phases of the development and evaluation of BIS have been published elsewhere [13,14].

\section{Method \\ Sample}

Although the BIS accepts referrals for patients with both malignant and non-malignant conditions, COPD patients make up the largest diagnostic group of referrals. In addition, a body of work (cited earlier) has begun to address the role of such interventions (albeit only in a clinic setting) in patients with malignancies. Therefore this Phase II trial focused on a homogeneous cohort of COPD patients, although BIS continued to deliver the intervention to patients with other diagnoses such as cancer and heart failure outside of the trial.

\section{RCT design}

A single-blinded fast-track pragmatic RCT design was used. Patients were randomised either to a fast-track group (FT), where they received the intervention immediately, or the control condition (the waiting list group; $\mathrm{WL}$ ), where they received the intervention after an eightweek period on a waiting list during which time they received standard care.

\section{Definition of intervention and standard care}

Table 1 defines the intervention that BIS provided, in terms of a minimum set of core interventions for nonmalignant patients at Phase II.

The control group received standard care for an eightweek period before receiving BIS. Our definition of standard care, in the context of this Phase II RCT, was: specialist outpatient appointments in secondary care (e.g. respira- 
Table I: Service model for the Breathlessness Intervention Service (BIS) for non-malignant patients at Phase II RCT (model date: I5/ 12/06: since revised)

\begin{tabular}{ll}
\hline Target patient group: & $\begin{array}{l}\text { Refractory dyspnoea - chronic breathlessness which is medically } \\
\text { optimally managed }\end{array}$ \\
\hline Referral: & Post, fax, electronic \\
\hline Assessment lead: & Clinical Specialist Physiotherapist \\
\hline BIS team: & $\begin{array}{l}\square \text { Clinical Specialist Physiotherapist: expert in three different disease } \\
\text { groups (cancer, heart failure, COPD), conducts highly specialised } \\
\text { assessment, works off-site and on-site. } \\
\square \text { Palliative Medical Consultant }\end{array}$ \\
\hline
\end{tabular}

\begin{tabular}{|c|c|}
\hline Medical assessment: & May be required \\
\hline Average no. of home visits: & 3 \\
\hline Average no. of telephone contacts: & 3 \\
\hline Ratio of face-to-face to telephone: & $1: 1$ \\
\hline Average length of service contact: & $6-8$ weeks \\
\hline \multirow[t]{3}{*}{ Outcome measures collected at first assessment: } & $\begin{array}{l}7 \text { modified Borg [19] at rest, self-reported, on exertion completion } \\
\text { of exercise test }\end{array}$ \\
\hline & $\begin{array}{l}\text { anxiety due to breathlessness at rest, self reported, on exertion \& } \\
\text { on completion of exercise test }\end{array}$ \\
\hline & $\begin{array}{l}\text { physiological measures e.g. oxygen saturation, heart rate \& } \\
\text { respiratory rate }\end{array}$ \\
\hline
\end{tabular}

\begin{tabular}{ll}
\hline Non-pharmacological interventions: & Ist stage of intervention \\
\hline Pharmacological interventions: & $2^{\text {nd }}$ stage of intervention \\
\hline Ist stage interventions (selection \& application as clinically indicated, & $\square$ explanation \& reassurance \\
majority used): & $\square$ anxiety management \\
& $\square$ psychological support \\
& $\square$ hand-held fan \\
& $\square$ information fact sheets \\
& $\square$ emergency plan \\
& $\square$ positioning to reduce work of breathing (rest, recovery \& activity) \\
& $\square$ breathing control \\
& $\square$ education to patient, carer \& health care generalists \\
& $\square$ pacing \& lifestyle adjustment \\
& $\square$ individualised exercise plan \\
& $\square$ relaxation \& visualisation \\
& $\square$ airway clearance techniques \\
& $\square$ advice regarding nutrition \& hydration \\
& $\square$ support to family \& patient to utilise education \& self-support \\
programmes & $\square$ sleep hygiene \\
& $\square$ smoking cessation prompt \\
& $\square$ brief cognitive therapy \\
& $\square$ pharmacological review
\end{tabular}


Table I: Service model for the Breathlessness Intervention Service (BIS) for non-malignant patients at Phase II RCT (model date: I5/ 12/06: since revised) (Continued)

\begin{tabular}{ll}
\hline $2^{\text {nd }}$ stage interventions (choice dependent on outcome of first stage & $\square$ further pharmacological review e.g. low dose opioids, anti- \\
$\begin{array}{ll}\text { interventions): } & \text { depressants, anxiolytics } \\
& \square \text { referral to specialist services (see below) } \\
& \square \text { referral for long term oxygen therapy (LTOT) or short burst } \\
\text { oxygen therapy (SBOT) assessment }\end{array}$
\end{tabular}

$\square$ individualised patient plan

discharge summary to referrer with copies to GP, specialist services the patient was already in contact with (e.g. respiratory physicians), other involved health care professionals

(e.g. district nurses, nursing home care staff)

Referrals: tory) that may include specialist nurse input, and primary care services.

\section{Single-blinding}

Various placebos were considered but found to be unworkable for the clinicians and unbelievable as 'interventions' to the patient and carer populations. It was also considered possibly unethical for the clinicians given that BIS was using evidence-based interventions. Further, it was impossible to blind the clinicians to the group to which patients were allocated as the providers delivered the intervention to both groups and had necessary access to their referral histories (i.e. dates of referral). In pragmatic trials of services, blinding patients and clinicians is nearly always impossible $[8,15]$. For this Phase II trial an attempt was made to blind the researcher to the allocation of respondents until the end of the intervention period (week 8).

This was achieved by the researcher conducting the recruitment to the study and collecting baseline measures, but then passing the process of randomisation and reporting of allocation over to a third party (Addenbrooke's clinical trials' team). Patients were randomised to the fasttrack group (FT) or the waiting list group (WL) by the clinical trials' team sequentially opening sealed opaque numbered envelopes containing the random group allocation previously generated by a computer programme at King's College London (KCL). The envelopes were set up by an administrator at KCL not associated with the study.

Patients were then informed of the outcome of randomisation by the clinical trials' team, by telephone. BIS was then notified of the outcome of randomisation by the clinical trials' team (by telephone and secure email) in order that the service could book the first appointment with the patient in-line with the study protocol. The researcher was then notified that randomisation had occurred, and that the patient and service had been informed, but not the outcome of the randomisation. The purpose and need for single blinding was explained to patients and carers at the recruitment visit and they were reminded at the start of each subsequent blinded interview to try not to let the researcher know their group allocation. In addition, all data were handled using study identity numbers; group allocation identifiers were only added at the analysis stage.

Data collected from the waiting list group once they were in receipt of BIS (after their period on the waiting list when their group allocation was blinded to the researcher) was treated as before/after data and not RCT data. This allowed for the collection of qualitative data at the midpoint of using the service for the waiting list group as the researcher was no longer blinded to their group allocation, nor was she required to be at this stage.

\section{Measurement points}

Quantitative and qualitative data were collected by interview for all respondents at baseline ( $\mathrm{t} 1)$, prior to randomisation. A flow chart depicting the follow up measurement points is given in Figure 1.

\section{Sample size}

As this was a feasibility study, comparative analysis was not our primary objective thus adequate powering of the 


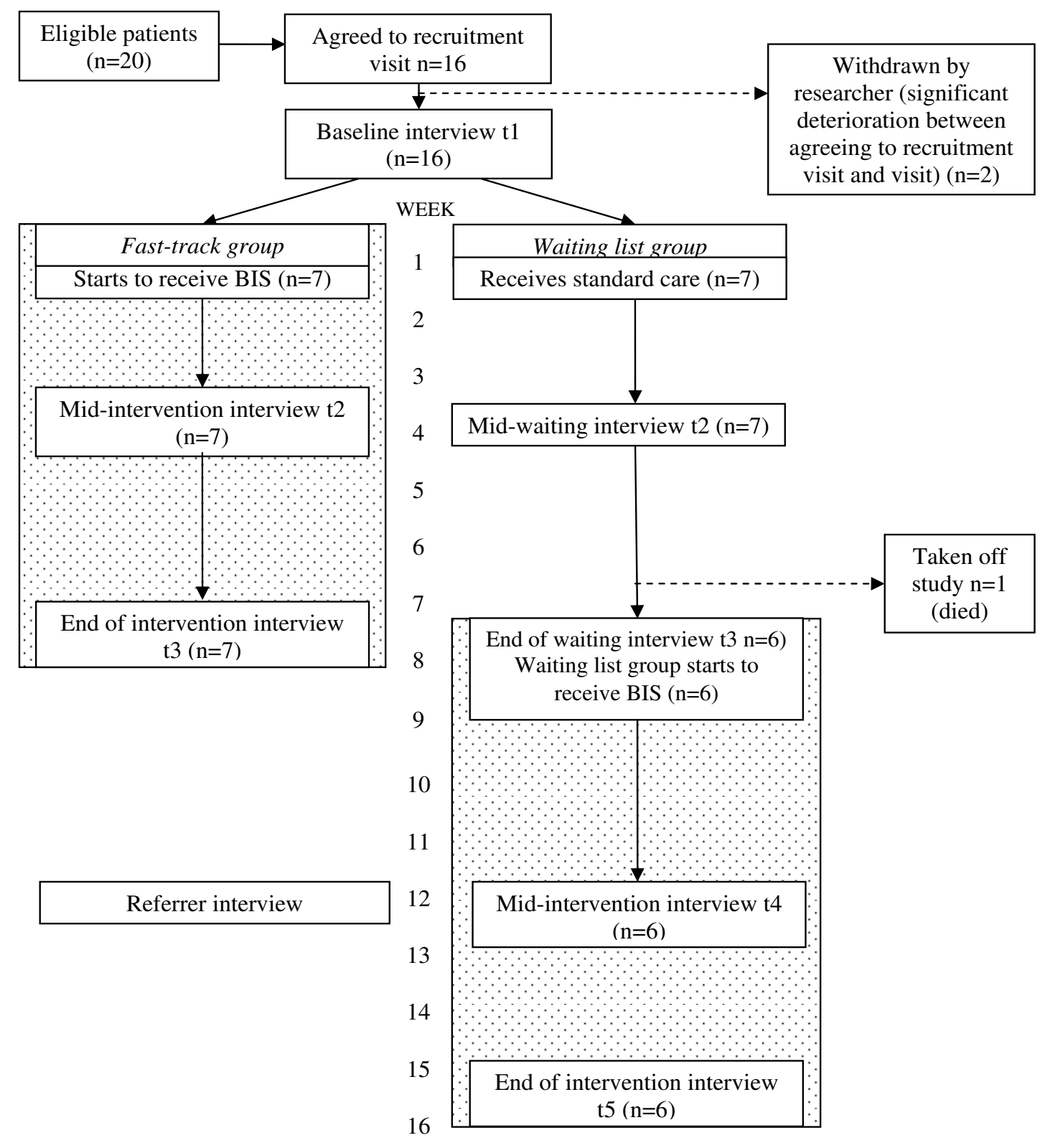

17

18

19

$20 \quad$ Referrer interview

Figure I

Flow chart depicting the follow up measurement points 
Table 2: Patient and carer baseline and outcome measures for BIS Phase II RCT

\begin{tabular}{|c|c|}
\hline Baseline characteristic/outcome & Instrument/measure \\
\hline \multicolumn{2}{|l|}{ PATIENT } \\
\hline Patient breathlessness & $\begin{array}{l}\text { Modified Borg [19] at rest and on exertion \& identification of activity that makes } \\
\text { breathlessness worst }\end{array}$ \\
\hline Patient breathlessness at best/worst & Visual Analogue Scale (VAS) [7] \\
\hline Patient functional ability & WHO performance scale [16] \\
\hline Patient social functioning & No. of times patient goes out of the house (average for last month) \\
\hline Patient quality of life & SEIQoL-DW [I7] \\
\hline Patient satisfaction with the service & Qualitative ( $\mathrm{tI}$ - expectations of the BIS; $\mathrm{t} 3 / \mathrm{t} 5$ - useful aspects of the service) \\
\hline Patient critical incidents data & $\begin{array}{l}\text { Qualitative } \\
\text { (e.g. identification of examples of difficulty getting help/medication/advice needed) }\end{array}$ \\
\hline Patient anxiety & Hospital Anxiety and Depression Scale [20] \\
\hline Patient distress due to breathlessness & Visual Analogue Scale for distress caused by breathlessness [7] \\
\hline Patient mastery of breathlessness & Mastery items from Chronic Respiratory Questionnaire [18]' \\
\hline Patient use of other services & Service use questions \\
\hline
\end{tabular}

\section{CARER}

Carer quality of life

Carer satisfaction with the service

Carer critical incidents data

Carer anxiety

Carer's assessment of patient's breathlessness

breathlessness worst

Carer's assessment of patient's breathlessness at best/worst VAS adapted for carer

Carer distress due to patient's breathlessness

Carer's assessment of patient's use of services

Carer's social functioning

Caregiver burden

Qualitative

Service use questions

Burden Interview [2I]

\section{SEIQoL-DW [17]}

Qualitative ( $\mathrm{t} I$ - expectations of the BIS; $\mathrm{t} 3 / \mathrm{t} 5$ - useful aspects of the service)

(e.g. identification of examples of difficulty getting help/medication/advice needed)

Hospital Anxiety and Depression Scale [20]

Modified Borg [19] at rest and on exertion \& identification of activity that makes

VAS adapted for carer - distress caused to carer by patient's breathlessness

No. of times carer goes out of the house (average for last month)

Caregiver Appraisal Scale [22]

'Confirmation that it was valid to use these items in isolation of the whole Chronic Respiratory Questionnaire was received from its developer, Gordon Guyatt (email communication, 2005). 
trial was not required. Based on clinical and research experience and data published from other studies we aimed to recruit a maximum of 28 patients to the trial. Phase II would then provide the data, at this time lacking, to inform the power calculations for a Phase III RCT.

\section{Baseline and outcome measures}

Baseline characteristics and outcomes included patients' breathlessness, patient and carer distress due to breathlessness, patient mastery of breathlessness, patient and carer quality of life, patient and carer anxiety, other service use, caregiver burden, and patient and carer expectations of and satisfaction with the service. Table 2 outlines the baseline and outcome measures selected following a review of the literature $[7,16-22]$. The resulting interview schedule was both quantitative and qualitative at each measurement point except $\mathrm{t} 2$; thus this was a mixed methods RCT.

Despite not powering the trial, a primary outcome measure was identified in order that the results could inform the sample size calculations for Phase III. The primary outcome measure was 'distress due to breathlessness' measured using a Visual Analogue Scale (VAS; 0-10 cm); a difference between the baseline and follow up measurements of $1 \mathrm{~cm}$ on this scale could be regarded as clinically significant for patients with intractable breathlessness [23].

\section{Inclusion and exclusion criteria}

Table 3 shows the inclusion and exclusion criteria. These criteria were established in order to ensure the representativeness of the study sample to the population who would receive the BIS outside of the trial.

\section{Data collection}

Recruitment, randomisation and baseline interviews were conducted over a ten-month period between late March 2006 and early January 2007; the final patient interview was conducted in March 2007. All measurement points were audio-taped with respondents' permission and were conducted by experienced researchers (MF and GE). All patient interviews were conducted in their own homes; interviews with carers were conducted in their own homes, at their place of work or at their patient's home according to preference. Wherever possible, patients and carers were interviewed separately. Careful attention was paid to need, in terms of fatigue.

\section{Data management}

Quantitative data (e.g. structured questionnaires) were entered into SPSS. Interviews $\mathrm{t} 1, \mathrm{t} 3$ and $\mathrm{t} 5$ were transcribed by an independent transcription company, checked for accuracy and anonymised. Transcripts were then imported into NUD*IST software to enable storage and organisation of the data for analysis.

\section{Approvals and trial registration}

Ethics approval was obtained from the Cambridge LREC and R\&D approval from Addenbrooke's R\&D and the relevant local Primary Care Trusts (REC reference no. 05/ Q0108/471). The trial was registered with ClinicalTrials.gov (NCT00711438).

\section{Data analysis}

In order to examine the feasibility of the trial methodology, the following were examined: response rates to the trial; response rates to the individual questionnaires and items; comments relating to the trial functioning made

Table 3: Inclusion \& Exclusion criteria for entry to BIS Phase II RCT

\begin{tabular}{ll}
\hline Inclusion criteria & \\
\hline Patients & $\begin{array}{l}\text { i) Appropriate referral to the BIS } \\
\text { ii) Diagnosis of COPD/COAD } \\
\text { iii) Aged I8 years or over } \\
\text { iv) Any patient who does not meet any of the exclusion criteria }\end{array}$ \\
\hline Carers & $\begin{array}{l}\text { i) The informal carers of patients specified above, who can be significant others, relatives, friends or neighbours } \\
\text { ii) Aged I8 years or over } \\
\text { iii) Any carer who does not meet the exclusion criteria }\end{array}$ \\
\hline Exclusion criteria & $\begin{array}{l}\text { i) Any patient/carer unable to give informed consent } \\
\text { ii) Any patient living outside of Cambridgeshire PCT, West Essex PCT, East \& North Hertfordshire PCT, or Suffolk PCT } \\
\text { iii) Any patient who has previously had access to BIS } \\
\text { iv) Any patient/carer who is demented or confused } \\
\text { v) Any patient/carer with learning difficulties } \\
\text { vi) Any patient/carer from other vulnerable groups (e.g. head injury, severe trauma, and mental illness) }\end{array}$
\end{tabular}


during interviews with patients, carers, referrers and BIS providers; and, researcher fieldwork notes.

\section{Results}

Our original sample size was 28 patients. As the Phase II exploratory RCT progressed it became clear that: 1) BIS for patients with COPD could be conducted over a shorter time period than eight-weeks; 2) BIS needed to be evaluated across the full disease spectrum it served (malignant as well as non-malignant diseases other than COPD); 3) the service model for BIS was different for malignant and non-malignant conditions due to the differing disease trajectories, such that separate RCT protocols for evaluation of BIS for malignant and non-malignant conditions would be required; 4) some of the outcome measures were unsuitable or could be improved upon; 5) there would be difficulty maintaining clinical equipoise for COPD patients for a Phase III definitive RCT if the exploratory RCT continued; 6) assessment of cost effectiveness was warranted. Thus Phase II was discontinued 10 months into the RCT. Further details on these points follow in this and the following discussion sections.

Table 4: BIS Phase II RCT response rates

\begin{tabular}{|c|c|c|c|}
\hline ID No. & Agreed to recruitment/baseline visit? & Recruitment visit completed? & Reason for non-response \\
\hline 001 & Yes & Yes & \\
\hline 002 & No & $\mathrm{n} / \mathrm{a}$ & Moving house \\
\hline 003 & Yes & Yes & \\
\hline 004 & Yes & Yes & \\
\hline 005 & Yes & Yes & \\
\hline 006 & Yes & Yes & \\
\hline 007 & Yes & $\begin{array}{l}\text { No - withdrawn by researcher \& referred } \\
\text { straight to BIS }\end{array}$ & $\begin{array}{l}\text { Significant deterioration between agreeing \& } \\
\text { baseline interview }(\mathrm{t} I)\end{array}$ \\
\hline 008 & No & $\mathrm{n} / \mathrm{a}$ & Admitted to ITU - too ill on discharge \\
\hline 009 & Yes & Yes & \\
\hline 010 & No & $\mathrm{n} / \mathrm{a}$ & None given \\
\hline 011 & Yes & Yes & \\
\hline 012 & No & $\mathrm{n} / \mathrm{a}$ & Too ill (nursing home resident) \\
\hline 013 & Yes & Yes & \\
\hline 014 & Yes & Yes (but carer unobtainable) & \\
\hline 015 & Yes (but died on waiting list; carer refused) & Yes (but died on waiting list; carer refused) & \\
\hline 016 & Yes & Yes & \\
\hline 017 & Yes & Yes & \\
\hline 018 & Yes & $\begin{array}{l}\text { No - withdrawn by researcher \& referred } \\
\text { straight to BIS }\end{array}$ & $\begin{array}{l}\text { Admission \& significant deterioration } \\
\text { between agreeing \& baseline interview }(\mathrm{tl})\end{array}$ \\
\hline 019 & Yes & Yes & \\
\hline 020 & Yes & Yes & \\
\hline
\end{tabular}


At the time of discontinuation, 20 patients had met the inclusion criteria for Phase II. No patients with COPD referred to BIS during the study period were excluded. Table 4 shows the response rates of the 20 eligible patients.

Sixteen of the 20 patients invited to participate agreed to a recruitment visit, 14 completed the recruitment visit and 13 went on to complete the RCT protocol. Two patients were withdrawn by the researcher between agreeing to the recruitment visit and the conduct of the recruitment visit (one following research-carer phone discussions between the two events, and one on arrival of the researcher at the recruitment visit). This occurred prior to informed consenting and randomisation, and was due to significant deterioration in the patients' health: one was admitted to hospital with an acute exacerbation and the other was clearly entering the terminal stage and would not have been able to participate (the carer had called the GP just prior to the researcher's arrival).
No difficulties were reported by the third party randomisation team (Addenbrooke's Clinical Trials Team) either in terms of managing the randomisation process, contacting patients, contacting BIS or contacting the researcher. Table 5 shows the outcome of randomisation.

Seven patients were randomised to the fast-track (FT) group and seven to the waiting list (WL) group. One patient died whilst on the waiting list so reducing the final WL sample size to six: this was the only patient lost to attrition. Figure 2 provides a CONSORT flow chart summarising the numbers for study enrolment, randomisation, allocation, follow up and analysis. Carers were identified for all 13 respondents however one could not be contacted due to a house move; 12 therefore participated and completed the carers' protocol.

As described earlier, the protocol was designed in order that the researcher could remain blinded to group allocation until the qualitative questions at $\mathrm{t} 3$ (week 8). In this

Table 5: BIS Phase II RCT randomisation outcome

\begin{tabular}{|c|c|c|c|c|}
\hline $\begin{array}{l}\text { Recruitment/randomisation } \\
\text { order }\end{array}$ & Recruitment/ID no. & Fast-Track group & Waiting List group & Unblinded early? \\
\hline I & 01 & I & & By patient at $\mathrm{t} 2$ \\
\hline 2 & 03 & & I & No \\
\hline 3 & 05 & 2 & & No \\
\hline 4 & 04 & & 2 & By patient \\
\hline 5 & 06 & & 3 & By patient at $\mathrm{t} 3$ (early) \\
\hline 6 & 09 & 3 & & No \\
\hline 7 & 11 & & 4 & No \\
\hline 8 & 13 & 4 & & No \\
\hline 9 & 15 (died pre t3) & & $(\mathrm{X})$ & No \\
\hline 10 & 14 & 5 & & By patient on phone pre $t 3$ \\
\hline II & 16 & 6 & & By patient at $\mathrm{t} 2$ \\
\hline 12 & 17 & & 5 & $\begin{array}{l}\text { By carer at } \mathrm{tl} \text { (conducted post-patient's } \\
\mathrm{tl} \text { and randomisation)* }\end{array}$ \\
\hline 13 & 19 & & 6 & No \\
\hline 14 & 20 & 7 & & No \\
\hline Total & & 7 & 6 & $6 / 13$ \\
\hline
\end{tabular}

* unblinded to wrong group 


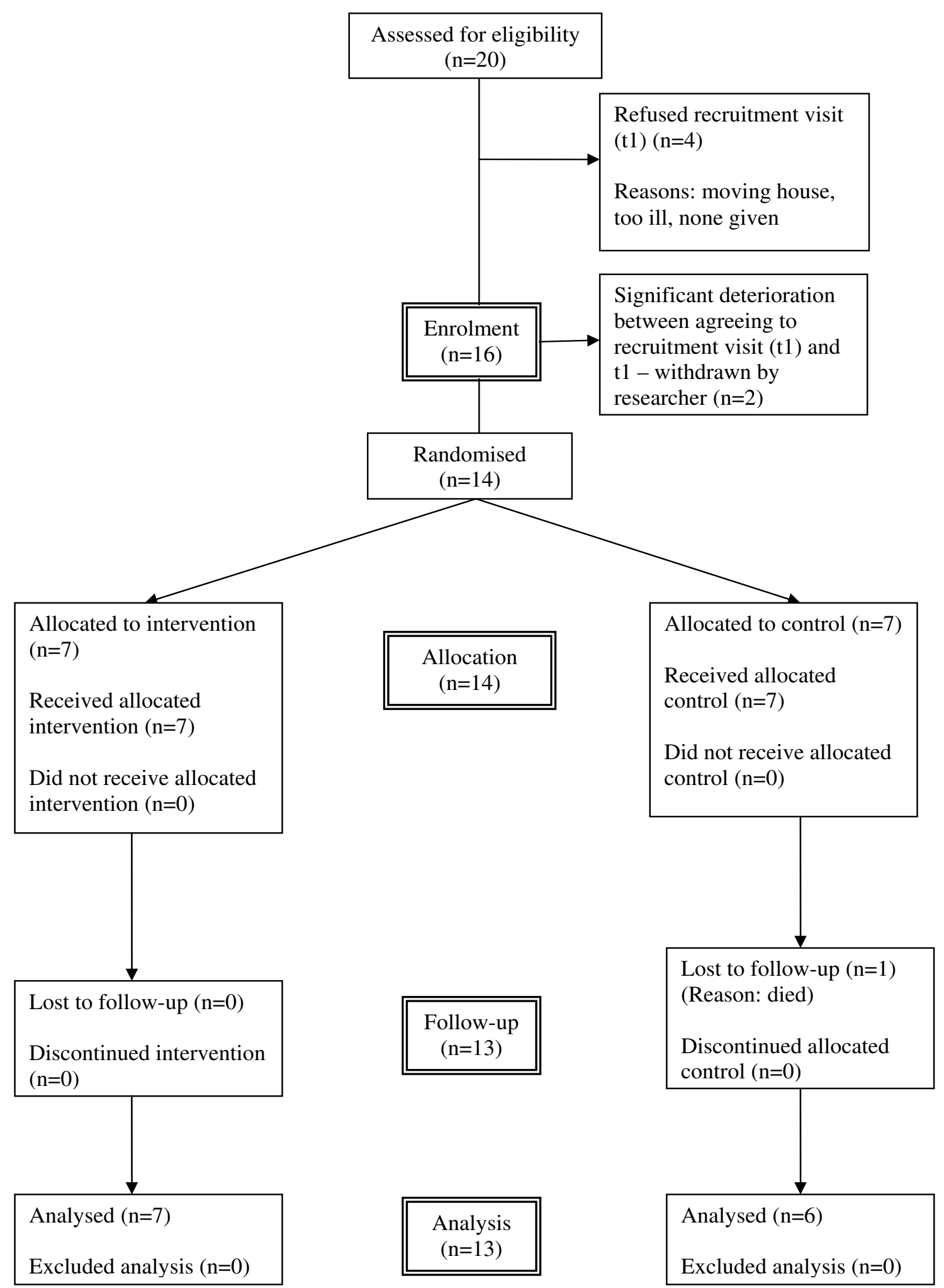

Figure 2

CONSORT flow chart summarising the numbers for study enrolment, randomisation, allocation, follow up and analysis 
pragmatic trial five of the 13 patients and one of the 12 carers who completed the study unblinded the researcher prior to this point (see Table 5). One of these unblindings (a carer) was to the wrong group i.e. the carer implied that their patient was in the fast-track group (receiving BIS) when in fact they were in the waiting list group and the patient had not yet seen BIS. Including this latter unblinding, there was therefore a 6 out of 25 respondents unblinding rate (base number consists of 13 patients and 12 carers). All unblindings appeared to be accidental rather than deliberate, and unblinders did not appear to be aware that they had unblinded themselves. Most unblindings took the form of mentioning a visit or contact by the BIS physiotherapist.

\section{Sample characteristics}

The age range of responding patients was 53-80 years, with a median of 69 years. The majority of patients were male (8/13). Their most recent FEV1 ranged from 0.681.28 litres/min and \% predicted ranged from $12.6-$ $28.9 \%$. The age range of responding carers was $29-76$ years, with a median of 57 years, and the majority of carers were female $(9 / 12)$.

Table 6: Obtained and missing data measurement points for patients and carers in BIS Phase II RCT

\begin{tabular}{|c|c|c|c|c|c|c|c|c|c|c|}
\hline \multirow[t]{3}{*}{ ID No. } & \multicolumn{10}{|c|}{ Interview no. } \\
\hline & \multicolumn{2}{|l|}{ tl } & \multicolumn{2}{|l|}{ t2 } & \multicolumn{2}{|l|}{ t3 } & \multicolumn{2}{|l|}{ t4 } & \multicolumn{2}{|l|}{ t5 } \\
\hline & $\mathbf{P t}$ & Carer & $\mathbf{P t}$ & Carer & $\mathbf{P t}$ & Carer & $\mathbf{P t}$ & Carer & $\mathbf{P t}$ & Carer \\
\hline 001 & $D$ & $\mathrm{D}$ & $D$ & $D$ & $D$ & $\mathrm{D}$ & $\mathrm{n} / \mathrm{a}$ & $\mathrm{n} / \mathrm{a}$ & $\mathrm{n} / \mathrm{a}$ & $\mathrm{n} / \mathrm{a}$ \\
\hline 003 & $\mathrm{D}$ & $\mathrm{D}$ & $\mathrm{D}$ & $\mathrm{D}$ & $\mathrm{D}$ & $\mathrm{D}$ & $\mathrm{D}$ & $\mathrm{D}$ & $\mathrm{D}$ & $\mathrm{D}$ \\
\hline 004 & $\mathrm{D}$ & D & $\mathrm{D}$ & $D$ & $\mathrm{D}$ & $D$ & $\mathrm{DI}$ & DI & D & $\mathrm{D}$ \\
\hline 005 & $\mathrm{D}$ & $\mathrm{D}$ & $\mathrm{D}$ & $D$ & $\mathrm{D}$ & $\mathrm{D}$ & $\mathrm{n} / \mathrm{a}$ & $\mathrm{n} / \mathrm{a}$ & $\mathrm{n} / \mathrm{a}$ & $\mathrm{n} / \mathrm{a}$ \\
\hline 006 & $D$ & $\mathrm{D}$ & $D$ & $D$ & $\mathrm{D}$ & $\mathrm{D}$ & $\mathrm{D}$ & DP & $\mathrm{D}$ & $\mathrm{D}$ \\
\hline 009 & $D$ & $\mathrm{D}$ & $D$ & $D$ & $D$ & $\mathrm{D}$ & $\mathrm{n} / \mathrm{a}$ & $\mathrm{n} / \mathrm{a}$ & $\mathrm{n} / \mathrm{a}$ & $\mathrm{n} / \mathrm{a}$ \\
\hline 011 & $D$ & $\mathrm{D}$ & $D$ & $D$ & $D$ & $\mathrm{D}$ & $\mathrm{D}$ & $\mathrm{D}$ & $\mathrm{D}$ & $D$ \\
\hline 013 & $D$ & $\mathrm{D}$ & $D$ & $D$ & $\mathrm{D}$ & $\mathrm{D}$ & $\mathrm{n} / \mathrm{a}$ & $\mathrm{n} / \mathrm{a}$ & $\mathrm{n} / \mathrm{a}$ & $\mathrm{n} / \mathrm{a}$ \\
\hline 014 & $D$ & $U$ & $D$ & $U$ & $D$ & $U$ & $\mathrm{n} / \mathrm{a}$ & $\mathrm{n} / \mathrm{a}$ & $\mathrm{n} / \mathrm{a}$ & $\mathrm{n} / \mathrm{a}$ \\
\hline 016 & $\mathrm{D}$ & $\mathrm{D}$ & $D$ & $\mathrm{D}$ & $\mathrm{D}$ & $\mathrm{D}$ & $\mathrm{n} / \mathrm{a}$ & $\mathrm{n} / \mathrm{a}$ & $\mathrm{n} / \mathrm{a}$ & $\mathrm{n} / \mathrm{a}$ \\
\hline 017 & $\mathrm{D}$ & $\mathrm{D}$ & $\mathrm{D}$ & $\mathrm{D}$ & $\mathrm{D}$ & $\mathrm{D}$ & DP & DP & $\mathrm{D}$ & $\mathrm{D}$ \\
\hline 019 & $D$ & $\mathrm{D}$ & $D$ & $x$ & $\mathrm{D}$ & DP & DP & DP & $\mathrm{D}$ & $\mathrm{D}$ \\
\hline 020 & $\mathrm{D}$ & $\mathrm{D}$ & $\mathrm{D}$ & $\mathrm{D}$ & $\mathrm{D}$ & $\mathrm{D}$ & $\mathrm{n} / \mathrm{a}$ & $\mathrm{n} / \mathrm{a}$ & $\mathrm{n} / \mathrm{a}$ & $\mathrm{n} / \mathrm{a}$ \\
\hline No. taped & 13 & 12 & 13 & 11 & 13 & 11 & 3 & 2 & 6 & 6 \\
\hline No. of interviews/total sought & $13 / 13$ & $12 / 13$ & $13 / 13$ & $11 / 13$ & $13 / 13$ & $12 / 13$ & $6 / 6$ & $6 / 6$ & $6 / 6$ & $6 / 6$ \\
\hline
\end{tabular}

Key:

$D=$ completed data collection point by interview and taped

$\mathrm{DI}=$ completed data collection point by interview, but not taped

DP = completed data collection point postally (at patient/carer request due to holiday), but not taped

$\mathrm{n} / \mathrm{a}=$ not applicable i.e. in fast-track group

$X=$ data collection point missed due to carer burden (family illness and other caring commitments)

$\mathrm{U}=$ carer unavailable due to house move 


\section{Patient and carer missing data}

For each patient and carer variable and outcome variable results are given in terms of the feasibility findings (missing data and comments on acceptability) for patients and for carers. Missed measurement points for patients and carers were minimal. As noted above, one carer could not be contacted at all due to a house move and one patient died before $\mathrm{t} 3$ and was therefore lost to attrition. The obtained and missing data points for the remaining 13 patients and 12 carers are given in Table 6.

For those completing the protocol Table 6 shows that there were no missed patient measurement points and only one missed carer measurement point: this was for a t2 interview for a carer whose caring role to the trial patient was secondary to another, primary, caring role: this measurement point was abandoned due to acute illness in the carer's family.

Quantitative patient and carer missing data for individual questionnaires and items is reported in Table 7 (Additional File 1).

\section{Trial methodology}

The fast-track trial methodology proved acceptable to the patients, carers, referrers and BIS providers. No complaints or negative comments were received from any participants randomised to the waiting list group about their period of waiting. The timings of the interviews (measurement points) were manageable from a researcher perspective, fitted with the service provision and appeared acceptable to respondents.

Patient and carer baseline and final interviews lasted between an hour and an hour and a half (approximately); other follow up interviews lasted between 30 minutes and an hour (approximately). Patients were contacted by telephone during the morning of each planned interview before the researcher set out, to re-check the suitability of the appointment in terms of the patients' health status that day: this resulted in a small number of interviews being re-scheduled.

\section{Service model}

In addition to these findings relating to the feasibility of the trial there were findings relating to the feasibility of the service model collected through interviews with the BIS staff themselves. The service model for Phase II was an eight-week intervention however it became clear that a more focused intervention could be conducted over a four-week period. This would have the added advantage of reducing the waiting time for patients randomised to the waiting list group to four-weeks in Phase III, so potentially increasing acceptability still further.

\section{Discussion \\ Response rate}

Although based on a small sample size, this Phase II RCT achieved a reasonably good response rate for a trial conducted in palliative care that is worthy of further comment. Possible reasons for this response rate include the relationship between the research and clinical team, the patient recruitment letter, the use of a fast-track design, the patient group, and patient altruism. The relationship between the research and clinical team was undeniably strengthened by the research-culture of the clinical team, in particular its desire to learn from the various phases of the evaluation and remodel the service accordingly. The evaluation was initiated by the lead clinician who founded the service (SB) and who invited in academic collaboration. The service has been involved in every phase of the evaluation but with all aspects of data collection and much of the analysis being conducted independently of it. This is similar to the participatory approach described by Hopkinson et al (2005) [24]. The frequently reported gate-keeping role of service providers [25-28] or lack of support from clinical colleagues [8] was therefore not an issue for this study.

A notable feature of the patient recruitment letter was that, at the ethics committee's insistence, it was printed on BIS's own clinical letterhead (which indicated it was part of the palliative care team), as opposed to on the academic letterhead of the independent research team (i.e. King's College London). This may have increased patients' acceptance of the trial.

Palliative care patients, and their families, can be reluctant to participate in trials with a traditional design, where patients are randomised to an intervention or no intervention [8]. The use of a fast-track design meant that all patients had access to the intervention and, in addition, those in the fast-track group had access to it earlier than would normally be the case. Within the context of NHS culture patients are familiar with waiting lists and often expect to wait for a service: thus the short lead-in time to receiving BIS for those randomised to the fast-track group may have increased the response rate to this study.

Further the patient group targeted by this study may in itself have had an impact on study recruitment. Services for COPD patients are inconsistent and sporadic $[13,29$ $31]$, and access to palliative care is limited [32-34]. Thus access to any service for these patients is likely to be desirable, and a novel service particularly so. This may have been further compounded by an appreciation of research focusing on a symptom that is distressing, yet not often discussed openly, as highlighted in the earlier phases of this research $[13,14,35]$. One Phase II patient described 
the symptom of breathlessness, unlike pain, as 'not very fashionable' (P006).

\section{Low attrition \& missing data rates}

The study achieved a low attrition rate and low missing data rate, again unusual for a palliative care RCT $[8,10]$. Using the same fast-track design Higginson et al (2008) [10] had lower still attrition and missing data rates: they suggested that the disease trajectory of the target group, combined with the use of a fast-track design and highly skilled interviewers were the likely explanations for their success; the same reasons are likely to hold true for this study. A similar study with patients with malignant disease may not achieve such relatively low rates. The small sample size negates the possibility of exploring the relationship between patient characteristics and the response rates, attrition or patterns of missing data.

\section{Single-blinding}

Our attempt at single-blinding was partially successful (6/ 25 early unblinding rate). This was achieved with relatively small effort on the part of the research, randomisation and clinical team. The effort on the part of respondents is unknown, but data collection was carefully designed to minimise this effort and maintain the quality of the respondent-interviewer relationship: all data collection prior to formal unblinding (all of $\mathrm{t} 2$ and first half of t3) was quantitative; formal unblinding occurred mid-t3 once all quantitative measures (including the primary outcome measure) other than service use data had been completed and before the qualitative interview; formal unblinding consisted of opening a sealed envelope in front of respondents, reading out the contents and asking respondents to confirm whether they had or had not received the service, it was therefore made clear that the researcher was now formally (and appropriately) unblinded and that they were free to report any contact with BIS or any other service in the subsequent 'use of services' questions and qualitative interview. Respondents were not therefore expected to maintain blinding whilst participating in qualitative interviews or answering questions about service use (of BIS or any other services). In the view of the experienced interviewer this strategy did not introduce distance between the respondent and interviewer, indeed respondents appeared to be encouraged at the 'scientific' nature of the study.

The source of the early unblinding was, in all cases, the respondents; this occurred most often at $\mathrm{t} 2$ when patients mentioned visits from named individuals. We recommend that the content of interviews at unblinded measurement points should be carefully considered when designing blinded-trial protocols: there is a need to find a balance between reducing the chances of accidental unblinding and the collection of data that is of value and that is blinded e.g. in the case of this study, primary outcome measure data was collected at each interview whilst the researcher was blinded but the collection of service use data and qualitative data was deferred until after the formal unblinding stage of the $\mathrm{t} 3$ interview.

\section{Baseline and outcome measures}

The majority of measures tested in the trial proved suitable. Key exceptions were the WHO Performance Scale [16] and the Schedule for the Evaluation of Individual Quality of Life-Direct Weighting (SEIQoL-DW) [17]. The WHO Performance Scale lacked specificity for advanced COPD patients and will be replaced in Phase III by the Australiamodified Karnosfsky Performance Status Scale (AKPS) [36]. Based on the Karnofsky Performance Scale (KPS) [37] and the community care based Thorne-modified KPS (TKPS) [38], the AKPS accommodates any setting of care and is considered more relevant to palliative care [36].

Despite the high completion rate on the SEIQoL-DW, concerns about aspects of the process of administration led the research team to question the validity of the results obtained [39]. In Phase III quality of life will be assessed using a combination of the EQ-5D [40] (a brief measure of health status/health-related quality of life also required for the cost-effectiveness analyses), the full Chronic Respiratory Questionnaire [18] (Phase II used only the mastery scale), and qualitative interviews (open questions on the impact of breathlessness and the BIS on quality of life). This in line with the recommendations of Guthrie et al (2001) [41] and the 2005 MRC Clinical Trials Unit/Cicely Saunders Foundation 'Improving Research Methodology in Breathlessness' meeting [7].

\section{Role of Phase II}

Grande \& Todd (2000) describe the need for feasibility studies and careful piloting in order to carry out successful RCTs in palliative care [8]. Conducting this Phase II RCT provided crucial process and outcome data that has subsequently informed the robust design of Phase III: a singleblinded fast-track pragmatic RCT of BIS versus standard care for patients with any diagnosis. In addition, Phase II has provided additional data to further refine the service model of BIS prior to the commencement of Phase III.

This feasibility study focused on patients with COPD. It seems reasonable, due to similarities in disease trajectories, to expect its findings (in terms of the feasibility and acceptability of the methodology) to be generalisable to patients with other non-malignant conditions with similar trajectories (e.g. cystic fibrosis) with intractable breathlessness who may be referred to BIS in Phase III. However, generalisability to patients with breathlessness due to malignancies or those non-malignant illnesses that follow 
a malignant trajectory (e.g. interstitial lung disease) is unknown.

While none of the individual components of this study could be described as particularly novel (e.g. fast-track methodology, single-blinding, secondary care intervention delivered in the community, RCT in palliative care), the combination is. Von Gunten (2005) highlights the need for 'well-powered definitive studies of both existing and new approaches in terminally ill patients with the most common symptoms', including breathlessness [42]. Further, Bausewein et al (2007), reporting on an international meeting on breathlessness, noted the need for further examination of breathlessness intervention services in order to elucidate which components work most effectively in different conditions (e.g. malignant and nonmalignant) [43]. Phase II has provided key data for the definitive Phase III RCT of BIS.

\section{Conclusion}

In conclusion, this Phase II RCT has provided valuable information of the acceptability and feasibility of an RCT of BIS for patients with COPD. It has shown that: singleblinding is possible in a palliative care RCT and highlighted aspects of protocol design to reduce the unblinding rate; fast-track trials appear acceptable for patients with advanced COPD, their carers and referrers, as well as the clinicians (intervention providers); high response rates and low attrition can be achieved in palliative care RCTs with non-malignant patients; and, that baseline and outcome measures could be improved on. The trial has also provided vital data for the sample size calculations for Phase III (ClinicalTrials.gov NCT006-78405; NCRN Portfolio Study No. 4829).

\section{List of abbreviations}

FEV1: forced expiratory volume in one second; NCRN: National Cancer Research Network.

\section{Competing interests}

$\mathrm{MF}$ and IJH declare that they have no competing interests.

SB (founder of service) and PF were the clinicians providing the intervention (Breathlessness Intervention Service).

\section{Authors' contributions}

MF co-designed Phase II, LREC and R\&D approval, main researcher on Phase II, analysed feasibility data, authored first and subsequent drafts of paper. IJH co-applied for Phase II funding, co-designed Phase II, contributed to the revising the paper. PF co-designed intervention, intervention provider (clinician), contributed to later drafts of the paper. SB founded service and evaluation (Pre-clinical phase), co-designed intervention, intervention provider (clinician), co-applied for Phase II funding, co-designed
Phase II, contributed to revising the paper. All authors read and approved the final draft.

\section{Additional material}

\section{Additional file 1}

Table 7 - Quantitative patient and carer missing data for individual questionnaires/items for BIS Phase II RCT. The data provided present quantitative patient and carer missing data for individual questionnaires and items.

Click here for file

[http://www.biomedcentral.com/content/supplementary/1472684X-8-9-S1.doc]

\section{Acknowledgements}

The authors would like to thank all participants who took part in and supported this study and this paper: the patients, carers, referrers and BIS staff; administrative staff at Addenbrooke's NHS Trust (namely Jacquie Adie \& Ann Cooke) and King's College London); Stephanie Vo (University of Cambridge); the Clinical Trials' Team for randomisation (Kelly Gleason and Roy Harris); Gail Ewing for locum interviewing; the funders (Gatsby Charitable Trust, Cicely Saunders International, Macmillan Cancer Support); and Professor Chris Todd.

\section{References}

I. Solano JP, Gomes B, Higginson IJ: A comparison of symptom prevalence in far advanced cancer, AIDS, heart disease, chronic obstructive pulmonary disease and renal disease. J Pain Symptom Manage 2006, 3 I (I):58-69.

2. Corner J, Plant H, A'Hern R, Bailey C: Non-pharmacological intervention for breathlessness in lung cancer. Palliat Med 1996, 10:299-305.

3. Bredin M, Corner J, Krishnasamy M, Plant H, Bailey C, A'Hern R: Multicentre randomised control trial of nursing intervention for breathlessness in patients with lung cancer. BMJ 1999, 318:901-904.

4. Hately J, Laurence V, Scott A, Baker R, Thomas P: Breathlessness clinics within specialist palliative care settings can improve the quality of life and functional capacity of patients with lung cancer. Palliat Med 2003, 17:410-417.

5. Connors S, Graham S, Peel T: An evaluation of a physiotherapy led non-pharmacological breathlessness programme for patients with intrathoracic malignancy. Palliat Med 2007, 2I(4):285-287.

6. MRC: A framework for development and evaluation of RCTs for complex interventions to improve health London: MRC; 2000.

7. Booth S: Improving research methodology in breathlessness: a meeting convened by the MRC Clinical Trials Unit and the Cicely Saunders Foundation. Palliat Med 2006, 20(3):219-220.

8. Grande GE, Todd CJ: Why are trials in palliative care so difficult? Palliat Med 2000, 14:69-74.

9. Higginson IJ, Vivat B, Silber E, Saleem T, Burman R, Hart S, Edmonds $P$ : Study protocol: delayed intervention randomised controlled trial within the Medical Research Council (MRC) Framework to assess the effectiveness of a new palliative care service. BMC Palliat Care 2006, 2(5):7.

10. Higginson IJ, Hart S, Burman R, Silber E, Saleem T, Edmonds P: Randomised controlled trial of a new palliative care service: compliance, recruitment and completeness of follow-up. BMC Palliat Care 2008, 7(I):7.

II. McWhinney IR, Bass MJ, Donner D: Evaluation of a palliative care service: problems and pitfalls. BMJ 1994, 309:1340-1342.

12. Hart T, Fann JR, Novack TA: The dilemma of the control condition in experience-based cognitive and behavioural treatment research. Neuropsychol Rehabil 2008, I8(I):I-2I. 
13. Booth S, Silvester S, Todd C: Breathlessness in cancer and chronic obstructive pulmonary disease: using a qualitative approach to describe the experience of patients and carers. J Palliat Support Care 2003, I(4):337-344.

14. Booth S, Farquhar M, Gysels M, Bausewein C, Higginson IJ: The impact of a breathlessness intervention service (BIS) on the lives of patients with intractable dyspnoea: a qualitative Phase I study. J Palliat Support Care 2006, 4:287-293.

15. Holloway E, Ram FSF: Breathing exercises for asthma. Cochrane Database Syst Rev 2004:CD00I 277.

16. World Health Organisation: WHO handbook for reporting results of cancer treatment. Geneva: WHO; WHO offset publication No. 48; 1979.

17. Hickey AM, Bury G, O'Boyle CA, Bradley F, O'Kelly FD, Shannon W: A new short form individual quality of life measure (SEIQoLDW): application in a cohort of individuals with HIVIAIDS. BMJ 1996, 3 I 3:29-33.

18. Guyatt GH, Berman LB, Townsend M, Pugsley SO, Chambers LW: A measure of quality of life for clinical trials in chronic lung disease. Thorax 1987, 42:773-778.

19. Burdon JGW, Juniper EF, Killian FE, Hargreave FE, Campbell EJM: The perception of breathlessness in asthma. Am Rev Respir Dis 1982 | 26:825-828.

20. Zigmond AS, Snaith RP: The Hospital Anxiety and Depression Scale. Acta Psychiatr Scand 1983, 67:361-370.

2I. Zarit SH, Reever KE, Bach-Peterson J: Relatives of the impaired elderly: correlates of feelings of burden. Gerontologist 1980, 20(6):649-655.

22. Lawton MP, Kleban MH, Moss M, Rovine M, Glicksman A: Measuring caregiving appraisal. J Gerontol 1989, 44(3):6I-7I.

23. Charles M, Reymond L, Israel F: Relief of incident dyspnea in palliative cancer patients: a pilot randomized, controlled trial comparing nebulized hydromorphone, systemic hydromorphone, and nebulized saline. J Pain Symptom Manage 2008, 36(I):29-38

24. Hopkinson JB, Wright DNM, Corner JL: Seeking new methodology for palliative care research: challenging assumptions about studying people who are approaching the end of life. Palliat Med 2005, 19:523-537.

25. Ewing G, Rogers M, Barclay S, McCabe J, Martin A, Todd C: Recruiting patients into a primary care based study of palliative care: why is it so difficult? Palliat Med 2004, 18:452-459.

26. Kendall M, Harris F, Boyd K, Sheikh A, Murray SA, Brown D, Mallinson I, Kearney N, Worth A: Key challenges and ways forward in researching the "good death": qualitative in-depth interview and focus group study. BMJ 2007, 334:52I-526.

27. White $C$, Gilshenan $K$, Hardy J: A survey of the views of palliative care healthcare professionals towards referring cancer patients to participate in randomized controlled trials in palliative care. Support Care Cancer 2008, I 6(I 2): I397-I405.

28. White CD, Hardy JR, Gilshenan KS, Charles MA, Pinkerton CR: Randomised controlled trials of palliative care - a survey of the views of advanced cancer patients and their relatives. European Journal of Cancer Care 2008, 44(13): 1820-1828.

29. Oliver SM: Living with failing lungs: the doctor-patient relationship. Fam Pract 200I, 18:430-439.

30. Elkington $\mathrm{H}$, White $\mathrm{P}$, Addington-Hall J, Higgs R, Pettinari C: The last year of life of COPD: a qualitative study of symptoms and services. Respir Med 2004, 98(5):439-445.

31. Elkington $\mathrm{H}$, White P, Addington-Hall J, Higgs R, Edmonds P: The healthcare needs of chronic obstructive pulmonary disease patients in the last year of life. Palliat Med 2005, 19:485-49I.

32. Skilbeck J, Mott S, Page H, Smith D, Hjelmeland-Ahmedzai S, Clark D: Palliative care assessment in chronic obstructive airways disease - a needs assessment. Palliat Med 1998, I 2:245-254.

33. Exley C, Field D, Jones L, Stokes T: Palliative care in the community for cancer and end-stage cardiorespiratory disease: the views of patients, lay-carers and health care professionals. Palliat Med 2005, 19:76-83.

34. Yohannes AM: Palliative care provision for patients with chronic obstructive pulmonary disease. Health Qual Life Outcomes 2007, 5:17.

35. Booth S: Improving the palliative care of patients with intractable breathlessness. In MD thesis London; University of London; 2009.
36. Abernethy A, Shelby-James T, Fazekas B, Woods D, Currow D: The Australia-modified Karnofsky Performance Status (AKPS) Scale: a revised scale for contemporary palliative care clinical practice. BMC Palliat Care 2005, 4:7.

37. Karnofsky D, Abelmann W, Craver L, Burchenal J: The use of nitrogen mustard in the palliative treatment of cancer. Cancer 1948, 1:634-656.

38. Nikoletti S, Porock D, Kristjanson LJ, Medigovich K, Pedler P, Smith M: Performance status assessment in home hospice patients using a modified form of the Karnofsky Performance Status scale. J Palliat Med 2000, 3:30I-3II.

39. Farquhar M, Higginson IJ, Booth S: Repeated measurement of quality of life in palliative care research: the experience of using the SEIQoL-DW in a pilot phase II RCT of a complex intervention for breathlessness [abstract]. Palliat Med 2008, 22(4):596.

40. The EuroQol Group: EuroQol-a new facility for the measurement of health-related quality of life. Health Policy 1990, 16(3): 199-208.

4I. Guthrie S], Hill KM, Muers MF: Living with COPD. A qualitative exploration of the experience of patients in Leeds. Respir Med 200I, 95:196-204.

42. Von Gunten CF: Interventions to manage symptoms at the end of life. J Palliat Med 2005, 8(sI):s-88.

43. Bausewein C, Malik F, Booth S, Higginson IJ: Recent developments in managing breathlessness: international researchers' meeting on Breathlessness in London, November 2006. Progress in Palliative Care 2007, I 5(6):279-284.

\section{Pre-publication history}

The pre-publication history for this paper can be accessed here:

http://www.biomedcentral.com/1472-684X/8/9/prepub

Publish with BioMed Central and every scientist can read your work free of charge

"BioMed Central will be the most significant development for disseminating the results of biomedical research in our lifetime. "

Sir Paul Nurse, Cancer Research UK

Your research papers will be:

- available free of charge to the entire biomedical community

- peer reviewed and published immediately upon acceptance

- cited in PubMed and archived on PubMed Central

- yours - you keep the copyright
BioMedcentral 